\title{
Molecular Detection of Glutenin and Gliadin Genes in the Domesticated and Wild Relatives of Wheat using Allele-specific Markers
}

\author{
J. Ahmadi ${ }^{1 *}$, A. Pour-Aboughadareh ${ }^{1}$, S. Fabriki-Ourang ${ }^{1}$ and A. A. Mehrabi ${ }^{2}$ \\ ${ }^{1}$ Department of Genetics and Plant Breeding, Imam Khomeini International University, Qazvin, Iran \\ ${ }^{2}$ Department of Agronomy and Plant Breeding, Ilam University, Ilam, Iran
}

(Received 30 May 2017; Accepted 8 November 2017;

Communicate by M. Molnár-Láng)

\begin{abstract}
Glutenin and gliadin subunits play a key role in flour processing quality by network formation in dough. Wild relatives of crops have served as a pool of genetic variation for decades. In this study, 180 accessions from 12 domesticated and wild relatives of wheat were characterized for the glutenin and gliadin genes with allele-specific molecular markers. A total of 24 alleles were detected for the Glu-A3 and Gli-2A loci, which out of 19 amplified products identified as new alleles. Analysis of molecular variance (AMOVA) indicated that 90 and $65 \%$ of the genetic diversity were partitioned within two Aegilops and Triticum genera and their species, respectively. Furthermore, all glutenin and gliadin analyzed loci were polymorphic, indicating large genetic diversity within and between the wild species. Our results revealed that allelic variation of Glu-3A and Gli-As.2 is linked to genomic constitutions so that, Ae. caudata (C genome), Ae. neglecta (UM genome), Ae. umbellulata (U genome) and T. urartu (A ${ }^{u}$ genome) harbor wide variation in the studied subunits. Hence, these species can be used in wheat quality breeding programs.
\end{abstract}

Keywords: Aegilops, Triticum, glutenin, gliadin, allelic diversity

\section{Introduction}

The presence of gliadins and glutenins are important factors in determining the viscoelastic and dough strength properties, which are responsible for bread-making qualities of wheat (Jin et al. 2011). Storage proteins in cereal grain comprise of two major types. In the first type, glutenin a polymer consisting of high-molecular-weight (HMW-GS) and low-molecular-weight subunits (LMW-GS), accounting for $60 \%$ of the storage protein in wheat endosperm. HMW-GS are coded by loci, Glu-A1, Glu-B1, and Glu-D1, on the long arms of chromosomes $1 A, 1 B$, and $1 D$, respectively, while LMW-GS are encoded using Glu-A3, Glu-B3, and Glu-D3, on the short arms of the same chromosomes (Jin et al. 2011). In the second type, gliadin made up of monomeric subunits (Payne 1987). The gliadin loci control the synthesis of a group of proteins, which they further were separated into three types: $\alpha / \beta$-gliadin, $\gamma$-gliadin and $\omega$-gliadin. The $\alpha / \beta$-gliadin subunits are located

\footnotetext{
*Corresponding author; E-mail: j.ahmadi@eng.ikiu.ac.ir
} 
on the Gli-2 loci of the short arm of the homoeologous group 6 chromosomes, while $\gamma$-gliadin, and $\omega$-gliadin are located on the Gli-1/Gli-3 loci of the short arm of the homoeologous group 1 chromosomes (Payne et al. 1984). Allelic variations of these protein compositions can result in functional differences, such as amino-acid substitutions and Indels (Ma et al. 2006), which may have an effect on dough quality.

Additionally to the variation found in improved modern lines, the search of new variation in wild relatives and alien species has great importance in expanding the wheat germplasm. Wild relatives are species that are closely related to crop plants, which can contribute useful traits such as biotic and abiotic resistance, as well as protein quality (Pour-Aboughadareh et al. 2017b, c). These species are of interest to plant breeders, and major efforts have been made to transfer their genetic variation into domesticated genotypes. The genera Aegilops and Triticum - belonging to the tribe Triticeae within the Pooideae subfamily of the grass family Poaceae - are important in wheat germplasm due to their evolutionary relationship with the major agricultural crop T. aestivum L (PourAboughadareh et al. 2017a). Kimber and Feldman (1987) and van Slageren (1994) described 22 Aegilops and five Triticum species, which have a wide repertoire of key alleles that can be used in wheat improvement programs. Previously, the analysis of storage proteins of the wild einkorn wheats by specific molecular markers and sodium-dodecylsulfate-polyacrylamide gel electrophoresis (SDS-PAGE) indicated the high levels of polymorphism (Caballero et al. 2008; Ahmadi and Pour-Aboughadareh 2015), which suggest these species could be sources of new alleles of the glutenin and gliadin genes. Many studies have been performed on the gliadin and glutenin variation of different Aegilops and Triticum species, e.g., Ae. cylindrica (Wan et al. 2002), Ae. longissima (Jiang et al. 2008), Ae. tauschii (Xu et al. 2010), T. urartu (Caballero et al. 2008; Ahmadi and Pour-Aboughadareh 2015; Cuesta et al. 2017), T. monococcum (An et al. 2006) and T. boeoticum (Ahmadi and Pour-Aboughadareh 2015). Despite prior studies showing that the wild relatives of wheat had the large variation in glutenin and gliadin subunits, no work has been undertaken to evaluate the whole samples of wild wheat species. With this in mind, the main goal of the current work was to charactererise the allelic variation of glutenin and gliadin in 180 accessions of Aegilops and Triticum collected from different regions of Iran using specific molecular markers. The results will benefit wheat quality improvement through marker-assisted selection.

\section{Materials and Methods}

\section{Plant Materials}

In total, 180 Aegilops and Triticum accessions from 12 domesticated and wild species were used for the characterization of glutenin and gliadin genes. They belonged to Ae. cylindrical Host., Ae. neglecta L., Ae. crassa Boiss., Ae. caudata L., Ae. speltoides Tausch., Ae. triuncialis, Ae. tauschii Coss. and Ae. umbellulata Zhuk.,T. boeoticum Boiss., T. urartu Gandilyan., T. durum Desf. and T. aestivum L., which collected from natural habitats, deserts, valleys and parts from the Zagros and central Elburz mountains 
located in the wide range from the north, northeast, northwest to southwest and central area of Iran. Detailed information about species' genomic constitution and eco-geographical distribution of these materials is listed in Table $\mathrm{S} 1 *$.

\section{DNA Extraction and PCR Amplification}

From each accession, the total genomic DNA was isolated according to the CTAB protocol (Doyle and Doyle 1987). The glutenin and gliadin specific primers used in this study were synthesized according to Long et al. (2005) and Kawaura et al. (2005), and their details are shown in Table S2. The PCR reaction was carried out in $15 \mu \mathrm{L}$ reaction mixtures contained $7.5 \mu \mathrm{L}$ master mix $(2 \mathrm{X}), 4.5 \mu \mathrm{L} \mathrm{ddH}_{2} \mathrm{O}, 2 \mu \mathrm{L}$ template DNA and $0.5 \mu \mathrm{L}$ of each forward/reverse primer pairs. Amplification was run at $94{ }^{\circ} \mathrm{C}$ for $5 \mathrm{~min}$, followed by 40 cycles of denaturation at $94{ }^{\circ} \mathrm{C}$ for 40 seconds, annealing at $53-58.3^{\circ} \mathrm{C}$ (varied for each primer) for 45 seconds and elongation at $72{ }^{\circ} \mathrm{C}$ for 90 seconds. The final extension was $7 \mathrm{~min}$ at $72{ }^{\circ} \mathrm{C}$. Amplified products were visualized on a $\%$ agarose gel, stained with safe view II and photographed under UV light.

\section{Data Analysis}

The alleles scored as 1 and 0 on the basis of presence and absence of each allele, respectively. The distribution of genetic variation within and among accessions and species (AMOVA), the percentage of polymorphic loci $(P P L)$, Nei's genetic diversity $(H)$ and Shannon's information $(I)$ indices was estimated using GenAlEX software version 6.5 (Peakall and Smouse 2006).

\section{Results}

\section{Frequency distributions of allelic variants in tested individuals and allelic diversity analysis}

Large differences in frequencies of detected alleles for glutenin and gliadin genes were observed in the tested accessions (Table 1). A total of 24 alleles were detected for the Glu$A 3$ and Gli-2A, which out of 19 amplified products identified as new alleles (Fig. 1). At Glu-3A locus, using the specific-locus marker Glu-3A.1, 43 (23.9\%), 24 (13.3\%), 21 (11.7\%) and 20 (11.1\%) accessions produced 400, 450, 500 and 600-bp alleles, respectively. Based on Glu-3A.2 marker, 9 (5\%), 2 (1.1\%), 3 (1.7\%), 1 (0.6\%), 128 (71.1\%), 2 $(1.1 \%), 2(1.1 \%)$ and $1(0.6 \%)$ accessions carried 150, 200, 250, 300, 350, 450, 500 and 800-bp alleles, respectively. Using Glu-3A.3, 3 (1.7\%), 1 (0.6\%), 20 (11.1\%), 10 (5.6\%), $111(61.7 \%)$ and $3(1.7 \%)$ individuals generated six alleles with the fragment size of 150 , $250,400,600$ and 680-bp, respectively. On the other hand, the frequency of the new alleles for Gli-As.2 locus was large. In the 180 tested accessions, 148 (82.2\%) individuals expressed an allele with size of 210-bp. The frequency of other amplified fragments by

\footnotetext{
*Further details about the Electronic Supplementary Material (ESM) can be found at the end of the article.
} 
AHMADI et al.: Molecular Detection of Glutenin and Gliadin Genes in the Domesticated

\begin{tabular}{|c|c|c|c|c|c|c|c|c|c|c|c|c|c|c|c|c|c|c|c|c|c|c|c|c|}
\hline \multirow{2}{*}{ 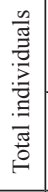 } & $\begin{array}{r}(\%) \\
\text { Кэuәnbə. }\end{array}$ & $\ddot{\tilde{\nu}}$ & $\stackrel{m}{=}$ & $\stackrel{.}{=}$ & $\exists$ & $\begin{array}{l}\circ \\
i\end{array}$ & $=$ & 羊 & $\stackrel{0}{0}$ & $\ddot{\nabla}$ & $\exists$ & 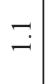 & $\begin{array}{l}0 \\
0 \\
0\end{array}$ & $\exists$ & $\begin{array}{l}0 \\
:\end{array}$ & $\exists$ & $\begin{array}{l}\stackrel{0}{i} \\
\dot{n}\end{array}$ & i. & $\underset{\infty}{\stackrel{N}{\infty}}$ & I & $\begin{array}{r}+ \\
\forall\end{array}$ & $\begin{array}{l}0 \\
i\end{array}$ & $\begin{array}{l}\hat{\infty} \\
\infty\end{array}$ & $\stackrel{\infty}{\sim}$ \\
\hline & oN & $\stackrel{\vartheta}{\sigma}$ & 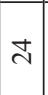 & $\vec{\sim}$ & $\stackrel{\sim}{ }$ & $a$ & $N$ & $m$ & -1 & $\stackrel{\infty}{\simeq}$ & $\sim$ & $\sim$ & - & $m$ & - & $\stackrel{\sim}{\circ}$ & 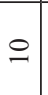 & $\Xi$ & $\stackrel{\infty}{ \pm}$ & $m$ & $\infty$ & $a$ & $\underset{1}{\sigma}$ & \pm \\
\hline \multirow{5}{*}{ 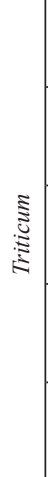 } & [170L & $\tilde{\sim}$ & $\stackrel{0}{\dot{m}}$ & $\ddot{2}$ & $\begin{array}{c}\mathfrak{c} \\
\infty \\
\sim \\
\sim\end{array}$ & $\stackrel{\circ}{\circ}$ & $\stackrel{0}{0}$ & $\because$ & $\stackrel{0}{\circ}$ & $\begin{array}{l}a \\
\infty \\
\infty\end{array}$ & $\ddot{0}$ & $\because$ & $\because$ & $\begin{array}{l}\infty \\
i\end{array}$ & $\stackrel{+}{-}$ & $\begin{array}{l}0 \\
i\end{array}$ & $\stackrel{-}{ \pm}$ & $\overrightarrow{0}$ & $\ddot{n}$ & $\stackrel{0}{\circ}$ & $\stackrel{\circ}{\circ}$ & $\stackrel{+}{-}$ & $\stackrel{0}{\circ}$ & $\stackrel{\sim}{+}$ \\
\hline & $\begin{array}{c}(\angle \mathrm{I}=\mathrm{U}) \\
\text { n...p..nn }\end{array}$ & $\hat{i}$ & $\because$ & 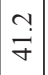 & $\stackrel{\circ}{\circ}$ & $\stackrel{0}{0}$ & $\because$ & $\stackrel{0}{0}$ & $\because$ & $\begin{array}{l}\stackrel{\nabla}{i} \\
\infty\end{array}$ & $\because$ & $\because$ & $\because$ & in & iे & 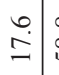 & $\begin{array}{l}\infty \\
\infty \\
\infty \\
i\end{array}$ & $\begin{array}{l}\infty \\
\infty \\
\infty \\
i\end{array}$ & $\begin{array}{l}c \\
\infty \\
\infty \\
\infty\end{array}$ & $\stackrel{\circ}{\circ}$ & $\stackrel{0}{\circ}$ & $\stackrel{0}{\circ}$ & $\begin{array}{l}0 \\
\dot{0}\end{array}$ & $\stackrel{0}{\circ}$ \\
\hline & $\begin{array}{r}(8 \mathrm{I}=\mathrm{u}) \\
\text { un.unp }\end{array}$ & $\ddot{0}$ & $\begin{array}{l}0 \\
\dot{8} \\
0\end{array}$ & $\because$ & $\begin{array}{l}0 \\
\dot{8}\end{array}$ & $\stackrel{\circ}{\circ}$ & $\because$ & $\stackrel{0}{0}$ & $\stackrel{\circ}{\circ}$ & $\begin{array}{l}+ \\
\dot{L}\end{array}$ & $\stackrel{\circ}{\circ}$ & $\because$ & $\because$ & $\because$ & $\stackrel{\circ}{\circ}$ & $\because$ & $\stackrel{0}{0}$ & $\begin{array}{l}a \\
\infty \\
\infty\end{array}$ & $\begin{array}{l}+ \\
\dot{\sigma}\end{array}$ & $\stackrel{\circ}{\circ}$ & $\stackrel{\circ}{\circ}$ & $\stackrel{0}{\circ}$ & 0 & $\stackrel{\circ}{\circ}$ \\
\hline & $\begin{array}{r}(\mathrm{LI}=\mathrm{U}) \\
\text { uпว!юоวоq }\end{array}$ & $\stackrel{\sim}{F}$ & $\because$ & 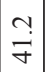 & $\stackrel{\circ}{\circ}$ & $\stackrel{0}{0}$ & $\because$ & $\because$ & $\stackrel{\circ}{\circ}$ & $\dot{8}$ & $\because$ & $\because$ & $\because$ & $\hat{n}$ & $\ddot{\circ}$ & $\dot{n}$ & $\ddot{0}$ & $\begin{array}{c}\vec{j} \\
i \\
\infty\end{array}$ & 守 & $\stackrel{\circ}{\circ}$ & $\stackrel{\circ}{\circ}$ & $\stackrel{0}{\circ}$ & $\begin{array}{l}0 \\
0\end{array}$ & $\because$ \\
\hline & 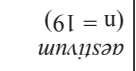 & $\because$ & $\overrightarrow{\dot{\lambda}}$ & $\because$ & $\because$ & $\because$ & $\because$ & $\because$ & $\stackrel{0}{\circ}$ & $\underset{\infty}{\infty}$ & $\stackrel{0}{\circ}$ & $\because$ & $\because$ & $\because$ & $\stackrel{\circ}{\circ}$ & $\because$ & $\stackrel{0}{0}$ & 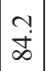 & İ & $\stackrel{0}{\circ}$ & $\begin{array}{ll}\circ & 1 \\
\circ & 4\end{array}$ & $\stackrel{m}{n}$ & $\stackrel{0}{0}$ & $\begin{array}{l}\infty \\
\ddot{n}\end{array}$ \\
\hline \multirow{9}{*}{ 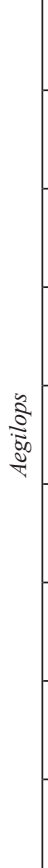 } & {$[\mathrm{E} 70 \mathrm{~L}$} & $\stackrel{\infty}{\dot{\sim}}$ & $\stackrel{\infty}{-}$ & 苛 & $\because$ & $\stackrel{m}{\infty}$ & $\stackrel{\infty}{\rightarrow}$ & $\begin{array}{l}\infty \\
i\end{array}$ & $\hat{\sigma}$ & $\frac{n}{3}$ & $\stackrel{\infty}{-}$ & $\stackrel{\infty}{-}$ & $\dot{o}$ & $\grave{0}$ & $\stackrel{0}{\circ}$ & $\stackrel{\sim}{ \pm}$ & $\stackrel{\circ}{\circ}$ & $\begin{array}{c}m \\
i \\
n\end{array}$ & $\begin{array}{l}\infty \\
\stackrel{2}{r}\end{array}$ & $\begin{array}{l}\infty \\
i\end{array}$ & $\stackrel{m}{?}:$ & $\stackrel{m}{r}$ & $\underset{ \pm}{ \pm}$ & $\overrightarrow{0}$ \\
\hline & $\begin{array}{r}(\angle \mathrm{I}=\mathrm{u}) \\
\text { płp|m|рqun }\end{array}$ & $\stackrel{\Upsilon}{g}$ & $\because$ & $\because$ & $\because$ & $\begin{array}{l}0 \\
\stackrel{0}{=}\end{array}$ & $\because$ & $\because$ & $\stackrel{0}{\circ}$ & Ұ̈ & $\stackrel{\infty}{\dot{\Xi}}$ & $\because$ & $\because$ & $\ddot{n}$ & $\stackrel{0}{0}$ & ت̇ & $\stackrel{\circ}{\circ}$ & $\because$ & ?n & $\stackrel{0}{\circ}$ & जे. & $\stackrel{0}{\circ}$ & $\begin{array}{l}0 \\
\dot{0}\end{array}$ & $\because$ \\
\hline & 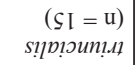 & 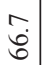 & $\because$ & $\because$ & $\stackrel{0}{0}$ & $\because$ & $\stackrel{m}{m}$ & $\because$ & $\stackrel{0}{\circ}$ & $\begin{array}{l}0 \\
\dot{\infty} \\
\dot{\infty}\end{array}$ & $\stackrel{0}{\circ}$ & $\because$ & $\because$ & $\because$ & $\because$ & $\because$ & $\stackrel{\circ}{\circ}$ & $m$ & $m$ & $\stackrel{\circ}{\circ}$ & $\begin{array}{lll}\circ & 1 \\
\circ\end{array}$ & $\hat{\sigma}$ & $\begin{array}{l}0 \\
\dot{0}\end{array}$ & $\stackrel{0}{\circ}$ \\
\hline & $\begin{array}{l}(0 \mathrm{z}=\mathrm{u}) \\
! ! y \rho s n \eta_{t}\end{array}$ & $\stackrel{0}{0}$ & $\begin{array}{l}0 \\
i n\end{array}$ & $\because$ & $\because$ & in & $\because$ & i. & $\because$ & $\begin{array}{l}\ddot{b} \\
\dot{f}\end{array}$ & $\because$ & $\because$ & $\stackrel{\circ}{0}$ & $\because$ & $\because$ & $\because$ & $\stackrel{0}{\circ}$ & $\ddot{8}$ & $\stackrel{0}{\dot{0}}$ & $\stackrel{\circ}{\circ}$ & $\begin{array}{l}: \\
\vdots\end{array}$ & $\begin{array}{lll}0 & 9 \\
i & & \end{array}$ & $\stackrel{0}{0}$ & $\stackrel{\circ}{\circ}$ \\
\hline & 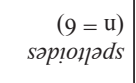 & $\because$ & $\because$ & $\because$ & $\stackrel{0}{\circ}$ & $\stackrel{0}{\circ}$ & $\because$ & $\stackrel{0}{0}$ & $\because$ & $\ddot{\circ}$ & $\stackrel{0}{\circ}$ & $\because$ & $\because$ & $\because$ & $\because$ & $\stackrel{0}{\circ}$ & $\stackrel{0}{\circ}$ & $\stackrel{m}{m}$ & $\underset{\infty}{\infty}$ & $\stackrel{\circ}{\circ}$ & $\stackrel{m}{m}$ & $\stackrel{0}{\circ}$ & $\begin{array}{l}0 \\
\dot{0}\end{array}$ & $\stackrel{\circ}{\circ}$ \\
\hline & $\begin{array}{l}(\mathrm{II}=\mathrm{u}) \\
p \neq \partial ว \mid \delta \partial u\end{array}$ & $\ddot{\dot{a}}$ & $\ddot{0}$ & $\begin{array}{l}0 \\
0\end{array}$ & $\stackrel{0}{\circ}$ & $\vec{a}$ & $\because$ & $\because$ & $\stackrel{\circ}{\circ}$ & $\hat{i}$ & $\stackrel{0}{0}$ & $\because$ & $\dot{o}$ & $\because$ & $\because$ & $\because$ & $\stackrel{0}{\circ}$ & $\frac{m}{\sim}$ & $\frac{\infty}{\dot{\infty}}$ & $\vec{a}$ & \begin{tabular}{l|l}
$n$ \\
$\vdots$ \\
$\dot{f}$
\end{tabular} & $\because$ & 帒 & $\ddot{n}$ \\
\hline & $\begin{array}{r}(6 \mathrm{I}=\mathrm{U}) \\
\text { pว!..pu! }{ }^{\circ}\end{array}$ & $\stackrel{\circ}{\circ}$ & ? & $\because$ & $\stackrel{\circ}{\circ}$ & $\because$ & $\because$ & $\stackrel{m}{n}$ & $\stackrel{m}{n}$ & $\tilde{\infty}$ & $\because$ & $\because$ & $\ddot{0}$ & $\because$ & $\because$ & $\because$ & $\stackrel{\circ}{\circ}$ & $\begin{array}{c}1 \\
\dot{\infty} \\
\dot{\infty}\end{array}$ & $\stackrel{\sim}{\stackrel{1}{\infty}}$ & $\stackrel{\circ}{\circ}$ & $\because$ & $\begin{array}{lll}\circ & 8 \\
0 & 8\end{array}$ & $\stackrel{0}{0}$ & $\begin{array}{l}\infty \\
i \\
i\end{array}$ \\
\hline & $\begin{array}{r}(\mathrm{t} \mathrm{I}=\mathrm{u}) \\
p S S D \cdot . l \partial\end{array}$ & $\stackrel{\circ}{\circ}$ & $\stackrel{\circ}{\circ}$ & $\stackrel{?}{\stackrel{m}{ \pm}}$ & $\because$ & $\stackrel{\vec{\Delta}}{\Delta}$ & $\stackrel{\circ}{\circ}$ & $\because$ & $\stackrel{0}{\circ}$ & $\begin{array}{l}\circ \\
\stackrel{0}{n}\end{array}$ & $\because$ & $\because$ & $\because$ & $\stackrel{0}{\circ}$ & $\stackrel{\circ}{\circ}$ & $\stackrel{0}{\circ}$ & $\stackrel{\circ}{\circ}$ & $\hat{\text { ă }}$ & 官 & $\vec{r}$ & 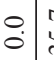 & $\begin{array}{ll}\hat{m} & \\
m & \end{array}$ & 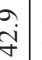 & $\stackrel{\circ}{\circ}$ \\
\hline & $\begin{array}{r}(L=\mathrm{u}) \\
\text { pұрpпn }\end{array}$ & $\stackrel{0}{\circ}$ & $\stackrel{0}{\circ}$ & $\stackrel{+}{\stackrel{\nabla}{~}}$ & $\stackrel{0}{\circ}$ & $\stackrel{0}{0}$ & $\because$ & $\stackrel{m}{ \pm}$ & $\stackrel{\circ}{\circ}$ & $\stackrel{0}{0}$ & $\stackrel{0}{\circ}$ & $\because$ & $\because$ & 0 & 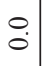 & $\stackrel{+}{i}$ & $\stackrel{\circ}{\circ}$ & $\because$ & $\stackrel{+}{\stackrel{\nabla}{*}}$ & $\stackrel{m}{+}$ & $\stackrel{1}{\circ}$ & $\stackrel{m}{ \pm}$ & $\stackrel{?}{ \pm}$ & $\stackrel{\circ}{\circ}$ \\
\hline & $\frac{0}{\frac{0}{\Xi}}$ & 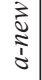 & 0 & $\begin{array}{l}\overrightarrow{3} \\
\stackrel{\vec{v}}{\vec{v}}\end{array}$ & $\tau$ & $\begin{array}{l}\frac{3}{\vec{v}} \\
\frac{\hat{v}}{0}\end{array}$ & $\mid \begin{array}{c}3 \\
\bar{v} \\
\hat{D}\end{array}$ & $\begin{array}{l}\vec{े} \\
\stackrel{\vec{े}}{\hat{v}}\end{array}$ & $\begin{array}{l}\frac{3}{0} \\
\frac{1}{0}\end{array}$ & $\circ$ & 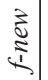 & 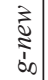 & 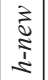 & 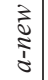 & \begin{tabular}{l} 
जे \\
\multirow{े}{1}{} \\
\end{tabular} & 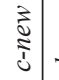 & $\frac{\overrightarrow{3}}{\frac{1}{0}}$ & 2 & $\sigma$ & है & $\begin{array}{l}\vec{z} \\
\stackrel{\vec{v}}{\vec{v}} .\end{array}$ & $\begin{array}{l}\frac{3}{0} \\
\frac{0}{0} \\
\frac{1}{0}\end{array}$ & 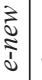 & 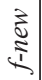 \\
\hline & $\stackrel{\mathscr{N}}{\tilde{n}}$ & ஓ & $\stackrel{\circ}{7}$ & \begin{tabular}{l}
8 \\
\hdashline
\end{tabular} & \& & 号 & 尺े & 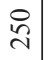 & ষ্ల & 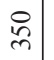 & 号 & 吕 & $\stackrel{\infty}{\infty}$ & $\stackrel{\circ}{2}$ & ڤ્) & ஓి & 8 & $\begin{array}{l}8 \\
\infty \\
0\end{array}$ & $\stackrel{\circ}{\sim}$ & in & 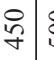 & 8 & 8 & $\gtreqless$ \\
\hline & 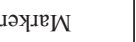 & & $\forall \mathcal{E}$ & $-n ! 1$ & & & & & $\forall \mathcal{E}^{-}$ & $-n 10$ & & & & & & $\forall \mathcal{E}^{-n} \eta_{1}^{1}$ & & & & & & 19 & & \\
\hline
\end{tabular}


this marker was small, so that $3(1.7 \%), 8(4.4 \%), 9(5 \%), 16(8.9 \%), 14(7.8 \%)$ and 4 $(2.2 \%)$ individuals generated fragments with 350, 450, 500, 600, 700 and 800-bp, respectively. In general, our results revealed that frequencies of new alleles in Aegilops are more than Triticum genus (Table 1). The result of analysis of molecular variance (AMOVA) showed that more than 90 and $65 \%$ of the genetic diversity was partitioned within two genera and different species, while the variation between them were 10 and $35 \%$

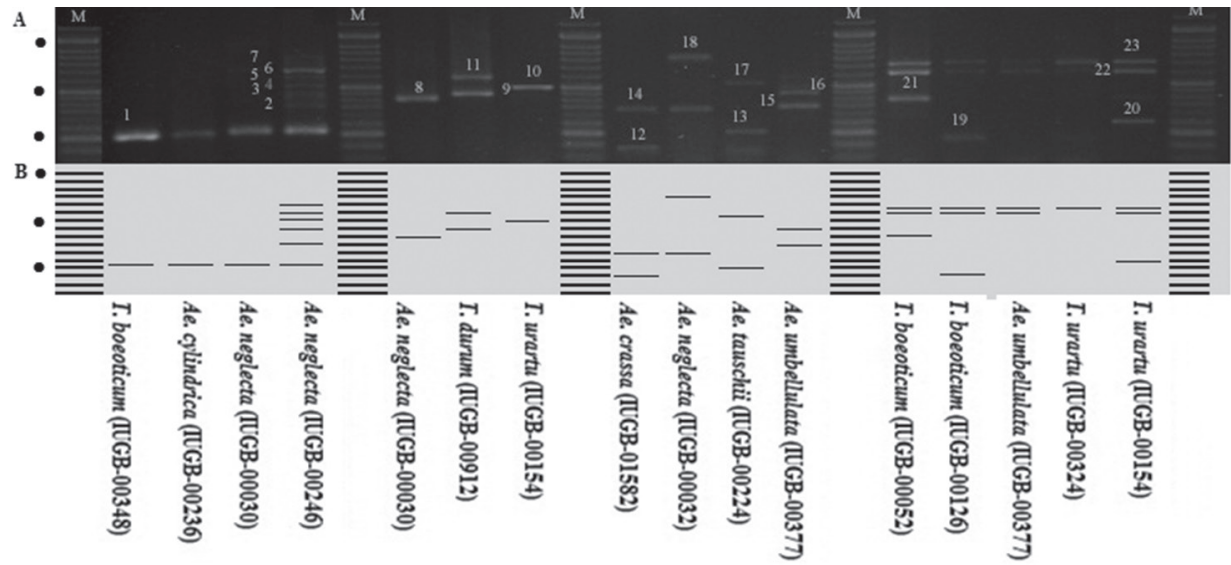

Figure 1. Panel A: image of agarose gel electrophoretic separation of the PCR products. Panel B: simulated electrophoretic image of the PCR products after normalization and thresholding. Each lane corresponds to different new alleles found. The molecular marker is a 50 bp Plus DNA ladder. In Panel B, dots label the 200, 500 and 1200 bp bands with stronger appearance in Panel A. M, ladder; 1, Gli-As.2a; 2, Gli-As.2b; 3, Gli-As.2c; 4, Gli-As.2d; 5, Gli-As.2e; 6, Gli-As.2f; 7, Gli-As.2g; 8, Glu-3A.1a; 9, Glu-3A.1b; 10, Glu-3A.1c; 11, Glu-3A.1d; 12, Glu-3A.2a; 13, Glu-3A.1b; 14, Glu-3A.1d; 15,Glu-3A.1e; 16,Glu-3A.1f; 17, Glu-3A.1g; 18, Glu-3A.1h; 19, Glu-3A.2a; 20,Glu-3A.2b; 21, Glu-3A.2c; 22, Glu-3A.2d; 23, Glu-3A.2e. For explanation of IUGB codes, see Table S1

Table 2. Analysis of molecular variance (AMOVA) in the wild relatives of wheat based on glutenin and gliadin markers

\begin{tabular}{|c|c|c|c|c|c|}
\hline Source & $d f$ & Sum of squares & Mean squares & $\begin{array}{l}\text { Estimated variance } \\
\text { components }\end{array}$ & Total variance $(\%)$ \\
\hline \multicolumn{6}{|c|}{ Genera } \\
\hline Among populations & 1 & 16.10 & 16.10 & 0.17 & 10 \\
\hline Within populations & 178 & 280.72 & 1.57 & 1.57 & 90 \\
\hline \multicolumn{6}{|c|}{ PhiPT $=0.097, P=0.010$} \\
\hline \multicolumn{6}{|c|}{ Species } \\
\hline Among populations & 11 & 110.95 & 10.08 & 0.60 & 35 \\
\hline Within populations & 168 & 185.87 & 1.11 & 1.11 & 65 \\
\hline \multicolumn{2}{|c|}{ PhiPT $=0.353, P=0.010$} & & & & \\
\hline
\end{tabular}


(Table 2). Also, the highest level of diversity and percentage of polymorphism loci ( $P P L)$ was observed in Aegilops than Triticum genus (87.50 vs. 58.33\%) (Table S3). Of the 12 different species, the highest values of $P P L, H$ and $I$ indices was estimated for Ae. neglecta followed by T. urartu, Ae. crassa, Ae. caudata and Ae. umbellulata.

\section{Distributions of detected alleles in the wild relatives of wheat}

Table 1 also presents the frequency of discovered alleles at loci analyzed in the domesticated and wild species of wheat. Additionally, allelic patterns observed by each accession are given in Table S4. As shown in Table 1, among the different species, only Ae. neglecta $(90.9 \%)$, Ae. triuncialis $(66.7 \%)$, T. urartu (52.9\%), T. boeoticum $(41.2 \%)$ and Ae. triuncialis (41.2\%) amplified the Glu-3A.1 a allele. All accessions of T. durum (100\%) along with some individuals of $T$. aestivum (21.1\%), Ae. cylindrica $(5.3 \%)$ and Ae. tauschii (5\%) possessed the Glu-3A.1b allele. T. boeoticum (41.2\%), T. urartu (41.2\%) Ae. caudata (71.4\%) and Ae. crassa (14.3) amplified the Glu-3A.1c. Moreover, only T. durum and some of individuals of T. aestivum presented amplification for Glu-3A.1d allele.

Ae. umbellulata, T. urartu, Ae. cylindrica, T. boeoticum and Ae. crassa carried the Glu-3A.1c allele by frequencies of $64.7,35.3,21.1,11.8$ and $7.1 \%$, respectively.

The frequency of Glu-3A.1d was high in Ae. triuncialis (100\%) and among other species only Ae. neglecta (18.2\%), Ae. crassa (14.3\%), Ae. tauschii (10\%) and T. boeoticum $(5.9 \%)$ presented amplification for this allele. Although the Glu3A-1e had the high frequency among some species, Ae. caudata, Ae. crassa, Ae. speltoides and T. boeoticum did not any amplify fragment for this allele. However, Ae. crassa and T. boeoticum along with Ae. triuncialis and Ae. tauschii produced the Glu-3A.1h allele (7.1, 11.8, 100 and 10\%, respectively).

According to allelic patterns revealed by Glu-3A.2 marker, some accessions belong to Ae. crassa (21.4\%), Ae. umbellulata (17.6\%), Ae. neglecta (9.1\%) and Ae. tauschii (5\%) amplified the Glu-3A.2a allele with 150-bp. Glu-3A.2b allele only presented in some accessions of Ae. triuncialis (13.3\%). The Glu-3A.2c allele also presented in some of Aegilops species such as Ae. caudata, Ae. cylindrica and Ae. tauschii with frequencies of $14.3,5.3$ and $5 \%$, respectively. The $G l u-3 A .2 d$, as a new allele, only was found in some of individuals of Ae. cylindrica (5.3\%). On the other hand, all species except Ae. caudata possessed the Glu-3A.2e allele and the highest frequency of it observed in T. boeoticum, T. durum, T. urartu and T. aestivum (100, 94.4, 82.4 and 78.9\%, respectively). Frequencies of Glu-3A.2f, Glu-3A.2g and Glu-3A.2h alleles were low and only Ae. umbellulata (11.8\%), Ae. tauschii (10\%) and Ae. neglecta $(0.9 \%)$ carried these alleles, respectively. Allelic frequencies revealed by Glu-3A.3 marker were similar to other glutenin markers. The highest frequency of Glu-3A.3a allele was found for Ae. tauschii, Ae. speltoides, Ae. neglecta and Ae. cylindrica $(65,16.7,9.1$ and 5.3\%, respectively). This allele was not observed in other species. The Glu-3A.3a allele was observed in T. urartu, T. boeoticum and Ae. umbellulata with frequencies of $5.9 \%$ for each species. The $G l u-3 A .3 b$ allele only observed in some T. urartu accessions (5.9\%). Among different species, Ae. caudata fol- 
lowed by Ae. umbellulata, T. urartu and T. boeoticum carried the Glu-3A.3c with frequencies of 71.4, 64.7, 17.6 and 5.9\%, respectively. Moreover, the Glu-3A.3d allele only was found in T. urartu with frequencies of $58.8 \%$. The Glu-3A.3e allele widely presented in all of the species (except Ae. umbellulata), and the highest frequencies was detected for Ae. crassa (92.9\%), T. durum (88.9\%), Ae. cylindrica (84.2\%), T. aestivum (84.2\%), respectively.

At the Gli-As locus, Gli-As.2a allele presented in all of species with high frequencies, while the Gli-As.2b allele only presented in Ae. caudata, Ae. crassa and Ae. neglecta. The Gli-As.2c allele mainly detected in some accessions of Ae. neglecta, Ae. speltoides (as the putative donor of B genome) and Ae. umbellulata with frequencies of 45.5, 33.3 and $5.9 \%$, respectively. Also, Ae. caudata (14.3\%), Ae. crassa (35.7\%), Ae. tauschii (5\%), Ae. triuncialis (6.7\%) and T. aestivum (5.3\%) possessed the Gli-As.2d. The Gli-As.2e only detected in Ae. neglecta, Ae. crassa, Ae. caudata and Ae. tauschii (with frequencies of 54.5, 42.9, 14.3 and 10\%, respectively). The Gli-As. $2 f$ allele mainly amplified in some of accessions of Ae. speltoides (50\%), Ae. neglecta (45.5\%), Ae. cylindrica (15.8\%) and T. aestivum (15.8\%). Furthermore, Gli.As. $2 g$ as the rare allele was only found in few accessions of Ae. caudata (14.3\%), T. aestivum (10.5\%) and Ae. neglecta (9.1\%).

\section{Discussion}

Glutenin and gliadin subunits play a crucial role in flour processing quality by network formation in dough. Due to this character, they allow wheat flour to be processed into bread, paste, noodles and other food products (Ma et al. 2006). Wild relatives and progenitors of wheat are of interest to plant breeders, and major efforts have been made to transfer their genetic variation into domesticated genotypes. These species have a wide repertoire of key alleles that can be used in wheat improvement programs (Pour-Aboughadareh et al. 2017a). In regard to storage proteins, extensive allelic diversity of glutenin and gliadin subunits have been discovered in several of wild relatives of wheat and previous studies have indicated that HMW glutenin subunits from different species of Aegilops such as Ae. tauschii have a significant influence on the processing quality of the synthetic hexaploid wheat (Zhang et al. 2013). As the major components of wheat storage proteins, LMW-GSs are significantly correlated with dough extensibility and dough strength (Zhang et al. 2012). In many studies, the correlations between allelic variations of Glu-3 and bread-making quality in common wheat are well established. Also, the linkage of specific LMW-GS proteins with all Glu-3 alleles makes it possible to discern the roles of individual LMW-GS in wheat flour functionality and develop new lines with improved quality (Lee et al. 2016). In the present study, we dissected the allelic variability of subunits of glutenin and gliadin in 180 accessions of wheat germplasm collected from different regions of Iran. According to results, all glutenin and gliadin loci analyzed were polymorphic, indicating large genetic diversity within and between species. Similarly, Ahmadpoor et al. (2014) and Ahmadi and Pour-Aboughadareh (2015) indicated that some of species of Aegilops and Triticum such as Ae. triuncialis, Ae. columnaris, Ae. biuncialis, Ae. crassa, Ae. cylindrica, Ae. ovate, T. boeoticum and T. urartu had high levels of 
allelic diversity in HMW and LMW subunits. Our results indicated that, the accessions from Aegilops have more allelic diversity than Triticum. Also, the results from AMOVA revealed a higher distribution of genetic variation within two genera and different species as compared to between them (Table 2). In addition, the highest percentage of polymorphism loci was detected in Aegilops than Triticum accessions. However, the highest values of Nie's genetic diversity $(H)$ and Shannon's information $(I)$ indices observed for Triticum accessions. Hence, these information could be useful to select material from these wild relatives for breeding purposes, because other interesting genes from different Aegilops and Triticum species could be also inherited and be present in various advanced wheat genotypes (Aguiriano et al. 2006; Ahmadi and Pour-Aboughadareh 2015).

More importantly, as shown in Table 1 and Fig. 1, we discovered 19 new allelic variants for Glu-3A and Gli-2A loci using four locus-specific primer pairs in the tested wheat germplasm. From species viewpoint, frequencies of new alleles in the various species were different. Regard to allelic status comparison, we surmise that present of new alleles in some of species linked to genomic constitution. For instance, Glu-3A.1a allele only presented in species that possessing U-genome, Ae. umbellulata (U genome), Ae. triuncialis (UC genome) and Ae. neglecta (UM genome), and Glu3A.1c linked to present of M genome in Ae. neglecta (UM) and Ae. crassa (MD). The Glu-3A.2c was associated with $\mathrm{D}$ and $\mathrm{C}$ genomes, so that Ae. cylindrica and its parental species, Ae. caudata (C genome) and Ae. tauschii (D genome), possessed this new allele. Furthermore, Glu-3Ac allele only exist in neglected diploid wheat; Ae. caudata (C genome), Ae. umbellulata (U genome), T. urartu ( $\mathrm{A}^{\mathrm{u}}$ genome) and T. boeoticum ( $\mathrm{A}^{\mathrm{b}}$ genome). Also, T. urartu, as A-genome donor of common wheat, carried two new alleles $G l u-3 A .3 c$ and $G l u-3 A .3 d$. In the present study, the number of allelic variants exceeded that found by Zhang et al. (2013) in common wheat, or by Wang et al. (2010), Ahmadpoor et al. (2014), Ahmadi and Pour-Aboughadareh (2015), Luo et al. (2015) and Cuesta et al. (2017) in wild relatives of wheat. The development of allele-specific markers is based on the polymorphisms of nucleotide sequences among different alleles, and the relationship between markers and phenotypes needs to be established. In this regard, Wang et al. (2009) discovered several new alleles in different wheat genotypes and designed seven PCR markers to discriminate the protein alleles $G l u-A 3 a, b, c, d, e, f$ and $g$. In another study, Wang et al. (2010) characterized one $G l u-3 A$ gene including four allelic variants, and developed a set of STS markers for the discrimination of the $G l u-3 A$ alleles.

Up to now, gliadin genes, not subjected to direct selection by wheat breeders, could be linked to genes directly selected from different wild relatives and ancestral species. Therefore, these new gliadin alleles might be associated with specific genomic constitution. The large number of new alleles indicates that some of the Aegilops and Triticum species are rather unique. This allelic diversity is conserved nowadays because wheat breeders did an important effort to collect and maintain this germplasm in ex-situ collections before being affected by the genetic erosion in the field (Aguiriano et al. 2006). Extensive researches have been performed to characterize novel gliadin genes among species of Aegilops and Triticum genera (Li et al. 2013; Zhang et al. 2015; Huang et al. 2016). In the present study, the Gli-As.2a allele was observed in all of Aegilops and Triti- 
cum species. Of the new alleles detected in Gli-As.2 locus, Gli-As.2d, e, $f$ and $g$ only discovered in T. aestivum, while these alleles were absent in other Triticum species. In contrast, allelic variability in Aegilops species was very high and some species had more than one new allele. The Gli-As.2b, $d$ and $e$ new alleles mainly were detected in Ae. caudata, Ae. crassa and Ae. neglecta. Also, Ae. neglecta possessed two new alleles Gli-As. $2 e$ and $f$. These results reveal that these species could be considered as valuable gene resources for seed storage proteins (Huang et al. 2016).

Wild relatives and progenitors of wheat are of interest to plant breeders, and major efforts have been made to transfer their genetic variation into domesticated genotypes. These species have a wide repertoire of key alleles that can be used in wheat improvement programs (Pour-Aboughadareh et al. 2017a). Of the wild relatives of common wheat, only some species such as Ae. tauschii, T. urartu, T. monococcum and T. dicocoides, have been explored and exploited for HMW and LMW characteristics. In conclusion, we reported an allelic diversity of glutenin and gliadin genes in different Aegilops-Triticum species that found a wide range of variability among various wild relatives of wheat using several locus-specific markers. These results can be used as a source of quality protein genes for wheat breeding and for studying the genetics of storage proteins. We also revealed that allelic status of Glu-3A and Gli-As. 2 is linked to genomic constitutions, so that the di- and tetraploid wild relatives possessing U, C, D and A genomes harbor wide variation in the glutenin and gliadin subunits. Hence, these results may open up new avenues for rethinking the connections between other progenitors and wild relatives with improving the dough quality. Consequently, it is suggested that the discovery of this highly diverse gene pool should encourage researchers to explore valuable and new alleles for the improvement of new varieties that are adapted to new uses.

\section{Acknowledgements}

The authors would like to acknowledge the Iran National Science Foundation (INSF) for the financial support of this work, through grant No. 94010881.

\section{References}

Aguiriano, E., Ruiz, M., Fite, R., Carrillo, J.M. 2006. Analysis of genetic variability in a sample of the durum wheat (Triticum durum Desf.) Spanish collection based on gliadin markers. Genet. Resour. Crop. Evol. 53:1543-1552.

Ahmadi, J., Pour-Aboughadareh, A. 2015. Allelic variation of glutenin and gliadin genes in Iranian einkorn wheat. Bio Env Sci. 7:168-179.

Ahmadpoor, F., Asghari-Zakaria, R., Firoozi, B., Shahbazi, H. 2014. Investigation of diversity in Aegilops biuncialis and Aegilops umbellulata by A-PAGE. Nat. Prod. Res. 28:1626-1636.

An, X., Zhang, Q., Yan, Y., Li, Q., Zhang, Y., Wang, A., Pei, Y., Tian, J., Wang, H., Hsam, S.L.K., Zeller, F.J. 2006. Cloning and molecular characterization of three novel LMW-i glutenin subunit genes from cultivated einkorn (Triticum monococcum L.). Theor. Appl. Genet. 113:383-395.

Caballero, L., Martin, M.A., Alvarez, J.B. 2008. Allelic variation for the high- and low-molecular-weight glutenin subunits in wild diploid wheat (Triticum urartu) and its comparison with durum wheats. Aust. J. Agric. Res. 59:906-910. 
Cuesta, S., Alvarez, J.B., Guzman, C. 2017. Identification and molecular characterization of novel LMW-m and -s glutenin genes, and a chimeric-m/-I glutenin gene in 1A chromosome of three diploid Triticum species. J. Cereal. Sci. 74:46-55.

Doyle, J.J., Doyle, J.L. 1987. A rapid DNA isolation procedure for small quantities of fresh leaf tissue. Phytochemical. Bulletin. 19:11-15.

Huang, Z., Long, H., Wei, Y.M., Yan, Z.H., Zheng, Y.L. 2016. Allelic variations of $\alpha$-gliadin genes from species of Aegilops section Sitopsis and insights into evolution of $\alpha$-gliadin multigene family among Triticum and Aegilops. Genetica. 144:213-222.

Jiang, C., Pei, Y., Zhang, Y., Li, X., Yao, D., Yan, Y., Ma, W., Hsam, S.L.K., Zeller, F.J. 2008. Molecular cloning and characterization of four novel LMW glutenin subunit genes from Aegilops longissima, Triticum dicoccoides and T. zhukovskyi. Hereditas. 145:92-98.

Jin, H., Yan, J., Pena, R.J., Xia, X.C., Morgounov, A., Han, L.M., Zhang, Y., He, Z.H. 2011. Molecular detection of high- and low-molecular-weight glutenin subunit genes in common wheat cultivars from 20 countries using allele-specific markers. Crop. Pasture. Sci. 62:746-754.

Kawaura, K., Mochida, K., Ogihara, Y. 2005. Expression profile of two storage-protein gene families in hexaploid wheat revealed by large-scale analysis of expressed sequence tags. Plant. Physiol. 139:1870-1880.

Kimber, G., Feldman, M. 1987. Wild wheat. An introduction. Special Report No. 353, Missouri, College of Agriculture, University of Missouri-Columbia.

Lee, J.Y., Beom, H.R., Altenbach, S.B., Lim, S.H., Kim, Y.T., Kang, C.S., Yoon, U.H., Gupta, R., Kim, S.T., Ahn, S.N., Kim, Y.M. 2016. Comprehensive identification of LMW-GS genes and their protein products in a common wheat variety. Funct. Integr. Genomics. 16:269-279.

Li, J., Wang, S.L., Cao, M., Lv, D.W., Subburaj, S., Li, X.H., Zeller, F.J., Hsam, S.L.K., Yan, Y.M. 2013. Cloning, expression, and evolutionary analysis of a-gliadin genes from and genomes. J. Appl. Genet. 54:157-167.

Long, H., Wei, Y., Yan, Z.H., Baum, B., Nevo, E. 2005. Composition, variation, expression and evolution of low-molecular-weight glutenin subunit genes in Triticum urartu. BMC Plant. Biol. 15:322.

Luo, G., Zhang, X., Zhang, Y., Yang, W., Li, Y., Sun, J., Zhan, K., Zhang, A., Liu, D. 2015. Composition, variation, expression and evolution of low-molecular-weight glutenin subunit genes in Triticum urartu. BMC Plant. Biol. 15: 322.

Ma, Z.C., Wei, Y.M., Long, H., Yan, Z.H., Baum, B., Zheng, Y.L. 2006. Characterization of low-molecularweight i-type glutenin subunit genes from diploid wheat in relation to the gene family structure. Mol. Biol. 40:897-906.

Payne, I.P., Jackson, E.A., Holt, L.M., Law, C.N. 1984. Genetic linkage between endosperm storage protein genes on each of the short arms of chromosomes 1A and 1B in wheat. Theor. Appl. Genet. 67:235-243.

Payne, P.I., Nightingale, M.A., Krattiger, A.F., Holt, L.M. 1987. The relationship between HMW glutenin subunit composition and the bread-making quality of British-grown wheat varieties. J. Sci. Food. Agr. 40:51-65.

Peakall, R., Smouse, P.E. 2006. GENALEX 6: genetic analysis in excel. Population genetic software for teaching and research. Mol. Ecol. Notes. 6:288-295.

Pour-Aboughadareh, A., Ahmadi, J., Mehrabi, A.A., Etminan, A., Moghaddam, M., Siddique, K.H.M. 2017 a. Physiological responses to drought stress in wild relatives of wheat: implications for wheat improvement. Acta. Physiol. Plant. 39:106.

Pour-Aboughadareh, A., Ahmadi, J., Mehrabi, A.A., Moghaddam, M., Etminan, A. 2017b. Evaluation of agromorphological diversity in wild relatives of wheat collected in Iran. J. Agr. Sci. Tech. 19:943-956.

Pour-Aboughadareh, A., Mahmoudi, M., Moghaddam, M., Ahmadi, J., Mehrabi, A.A., Alavikia, S.S. 2017 c. Agro-morphological and molecular variability in Triticum boeoticum accessions from Zagros Mountains, Iran. Genet. Resour. Crop. Evol. 64:545-556.

Van Slageren, M.W. 1994. Wild wheats: a monograph of Aegilops L. and Amblyopyrum (Jaub. and Spach) Eig (Poaceae). Wageningen Agricultural University Papers. Wageningen, The Netherlands.

Wan, Y., Wang, D., Shewry, P.R., Halford, N.G. 2002. Isolation and characterization of five novel high molecular weight subunit of glutenin genes from Triticum timopheevi and Aegilops cylindrica. Theor. Appl. Genet. 104:828-839. 
Wang, L., Li, G., Penea, R.J., Xia, X., He, Z. 2010. Development of STS markers and establishment of multiplex PCR for Glu-A3 alleles in common wheat (Triticum aestivum L.). J. Cereal. Sci. 51:305-312.

Wang, L.H., Zhao, X.L., He, Z.H., Ma, W., Appels, R., Peña, R.J., Xia, X.C. 2009. Characterization of lowmolecular-weight glutenin subunit Glu-B3 genes and development of STS markers in common wheat (Triticum aestivum L.). Theor. Appl. Genet. 118:525-539.

Xu, S.S., Khan, K., Klindworth, D.L., Nygard, G. 2010. Evaluation and characterization of high-molecular weight $1 \mathrm{D}$ glutenin subunits from Aegilops tauschii in synthetic hexaploid wheats. J. Cereal. Sci. 52:333336.

Zhang, X., Liu, D., Zhang, J., Jiang, W., Luo, G., Yang, W., Sun, J., Tong, Y., Cui, D., Zhang, A. 2013. Novel insights into the composition, variation, organization, and expression of the low-molecular-weight glutenin subunit gene family in common wheat. J. Exp. Bot. 64:2027-2040.

Zhang, X.F., Jin, H., Zhang, Y., Liu, D.C., Li, G.Y., Xia, X.C., He, Z.H., Zhang, A.M. 2012. Composition and functional analysis of low-molecular-weight glutenin alleles with Aroona near-isogenic lines of bread wheat. BMC Plant Biol. 12:243.

Zhang, Y., Luo, G., Liu, D., Wang, D., Yang, W., Sun, J., Zhang, A., Zhan, K. 2015. Genome, transcriptomeand proteome-wide analyses of the gliadin gene families in Triticumurartu. PLoS One. 10:e0131559.

\section{Electronic Supplementary Material (ESM)}

Electronic Supplementary Material (ESM) associated with this article can be found at the website of CRC at https://akademiai.com/loi/0806

Electronic Supplementary Table S1. List of the Aegilops - Triticum accessions analyzed, their provenance and genome classification

Electronic Supplementary Table S2. PCR-primers of molecular markers used for the detection of allelic diversity of glutenin and gliadin genes

Electronic Supplementary Table S3. Estimated genetic diversity parameters in Aegilops and Triticum species

Electronic Supplementary Table S4. Allelic patterns for gluten and gliadin genes in 180 Aegilops and Triticum accessions 